\title{
Eficacia del oxígeno hiperbárico en el tratamiento de la radionecrosis y el edema cerebral sintomáticos tras radiocirugía con acelerador lineal
}

\author{
M.A. Pérez-Espejo; R. García-Fernández*; B.M. Tobarra-González**; J.D. Palma-Copete**; A. González-López**; I. De \\ la Fuente-Muñoz*; J. Salinas-Ramos*; M. Felipe-Murcia; J.F. Martínez-Lage; J. Fernández-Pérez* y J.M. Romero***
}

Servicios de Neurocirugía. Oncología Radioterápica*, Radiofísica y Protección Radiológica**. Hospital Universitario "Virgen Arrixaca". Murcia. Cámara hiperbárica de Cartagena***. Hospital de Caridad. Cartagena. Murcia. España.

\section{Resumen}

La radionecrosis y el edema cerebral son complicaciones asociadas a la radiocirugía. Presentamos los casos de tres pacientes tratadas con radiocirugía mediante acelerador lineal, de un meningioma de polo frontal izquierdo, peñasco y parasagital parietooccipital, respectivamente, que desarrollaron, entre dos y ocho meses más tarde, lesiones de tipo radionecrótico con extenso edema peritumoral que resultaron resistentes al tratamiento con esteroides y que se han resuelto con la administración de $\mathbf{4 0}$ sesiones de oxígeno hiperbárico. Son pocos los casos publicados hasta ahora en la literatura con tan excelentes resultados, por lo que consideramos un hecho a tener muy en cuenta ante las posibles complicaciones de este tipo que puedan presentarse en el transcurso de la práctica radioquirúrgica antes de recurrir a la cirugía.

PALABRAS CLAVE: Radiocirugía. Radionecrosis. Oxígeno hiperbárico. Edema cerebral. Acelerador lineal

Usefulness of hyperbaric oxygen in the treatment of radionecrosis and symptomatic brain edema after LINAC radiosurgery

\section{Summary}

Radionecrosis with brain edema is a complication of radiosurgery. Three female patients harbouring a frontal pole, petrous and parasagital parietoocipital meningiomas respectively who had been treated with LINAC radiosurgery are presented. Those patients developed, between two and eight months later, a severe symptomatic radionecrosis with a huge brain edema resistant to the usual steroid therapy. Only after $\mathbf{4 0}$ sessions of hyperbaric oxygen, a good remission of the lesions was obtained. There are few cases reported in the literature with such a good outcome. Consequentely,

Recibido: 15-09-08. Aceptado 6-02-09 this therapy must be taken into account to treat this type of radiosurgical complication before considering surgery.

KEY WORDS: Radiosurgery. Radionecrosis. Hyperbaric oxygen. Brain edema. LINAC

\section{Introducción}

La radionecrosis es una complicación grave que puede desarrollarse tras el tratamiento de tumores o malformaciones vasculares intracraneales con radioterapia externa o radiocirugía. No existe, hasta el momento, una pauta a seguir, universalmente aceptada, para evitar este efecto adverso, si bien se ha hecho hincapié en la reducción del volumen de tejido normal irradiado como elemento de "seguridad", en el refinamiento de la conformación y de la homogeneidad del tratamiento radioquirúrgico y en otros aspectos que revisaremos más adelante. Desde el punto de vista terapéutico, sigue siendo válida la administración de esteroides u otros antioxidantes anticitoquinas para disminuir el edema cerebral circundante al tejido necrótico, la dieta baja en ion $\mathrm{Fe}$, así como la heparina por su efecto anticoagulante y antiinflamatorios sobre el endotelio ${ }^{17}$. La cirugía, en los casos incontrolables médicamente, es el último recurso a emplear para eliminar la lesión ocupante de espacio que la necrosis y su correlato edematoso suponen $^{13}$.

Alternativamente se ha invocado el efecto terapéutico del oxígeno hiperbárico, tanto en adultos como en niños ${ }^{3}$ que han sido radiados por diversas causas y que han desarrollado radionecrosis que, en ciertos casos, son graves e incluso letales.

Aportamos tres nuevos casos de radionecrosis cerebral sintomática, tras un tratamiento con radiocirugía, que han sido satisfactoriamente resueltos mediante una combinación de esteroides, oxígeno hiperbárico y heparina de bajo peso molecular. Creemos, dada la evolución de los casos que presentamos, que el papel estelar en esta multiterapia lo desempeña el oxígeno hiperbárico, pues no fue sino hasta su aplicación, cuando el cuadro clínico y la iconogra- 


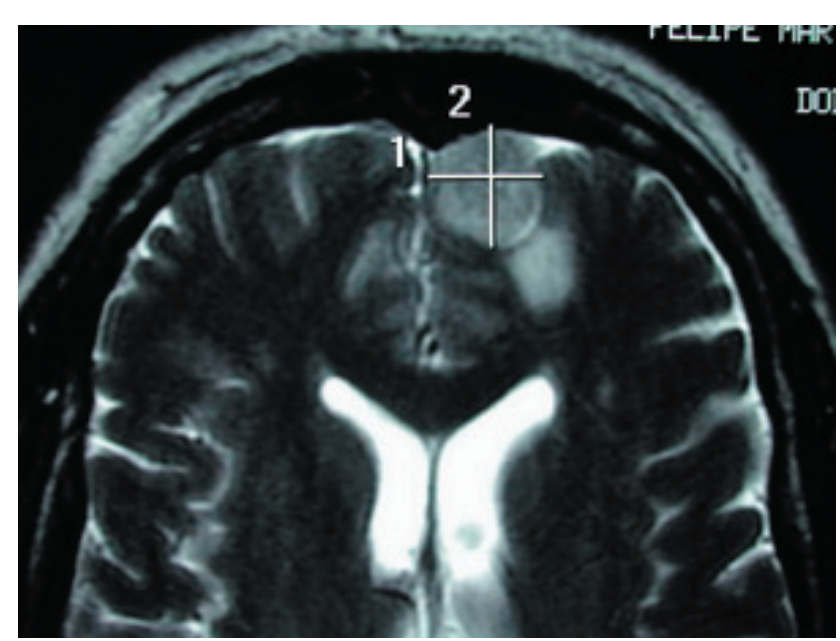

Figura 1.

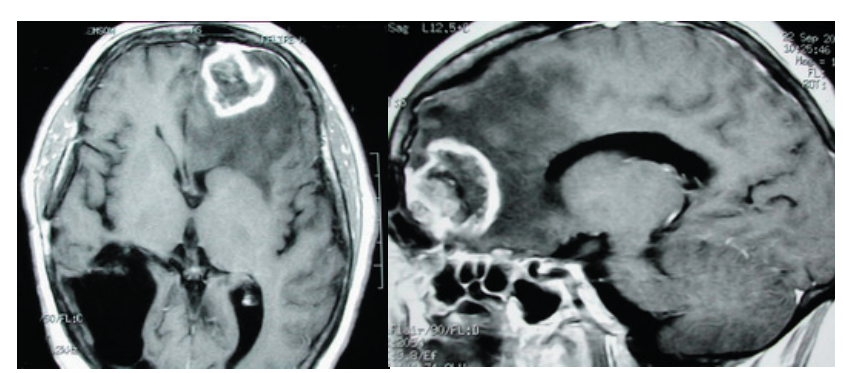

Figura 3.

fía comenzaron a mostrar una significativa mejoría.

\section{Material}

\section{$\operatorname{Caso} n^{\circ} 1$}

Mujer de 61 años, ya intervenida en 1996 de un meningioma parietal derecho. Con posterioridad comenzó a sufrir crisis comiciales generalizadas que fueron controladas satisfactoriamente con Fenitoína. En la exploración clínica hallamos una enferma obesa, con Karnofsky de 100 y una hemianopsia homónima izquierda desde el postoperatorio anterior. En la RNM rutinaria de control realizada en marzo 06 se halló un meningioma del polo frontal izquierdo, esférico, de unos 18 x $20 \mathrm{~mm}$ de diámetros máximos y con un volumen de 6,57 cc. (Fig. 1).

Se le ofrecieron diversas opciones terapéuticas, decidiéndose por la radiocirugía. Ésta se llevó a cabo el pasado 18-5-06 (Fig. 2)

Se realizó nueva RNM craneal con cortes finos y bajo anestesia local se colocó marco de Brain Lab y se hizo TAC craneal con localizador en condiciones estereotácticas. Se transfirieron los datos al programa Brain Scan $5.0 \mathrm{y}$, tras fusión de imagen de TAC y RNM, se efectuó el tratamiento usando un acelerador lineal Elekta Precise (6 MeV), con los siguientes parámetros: isocentros: 1; arcos: 5; colimador: $30 \mathrm{~mm}$; dosis de prescripción al isocentro: 18Gy; dosis
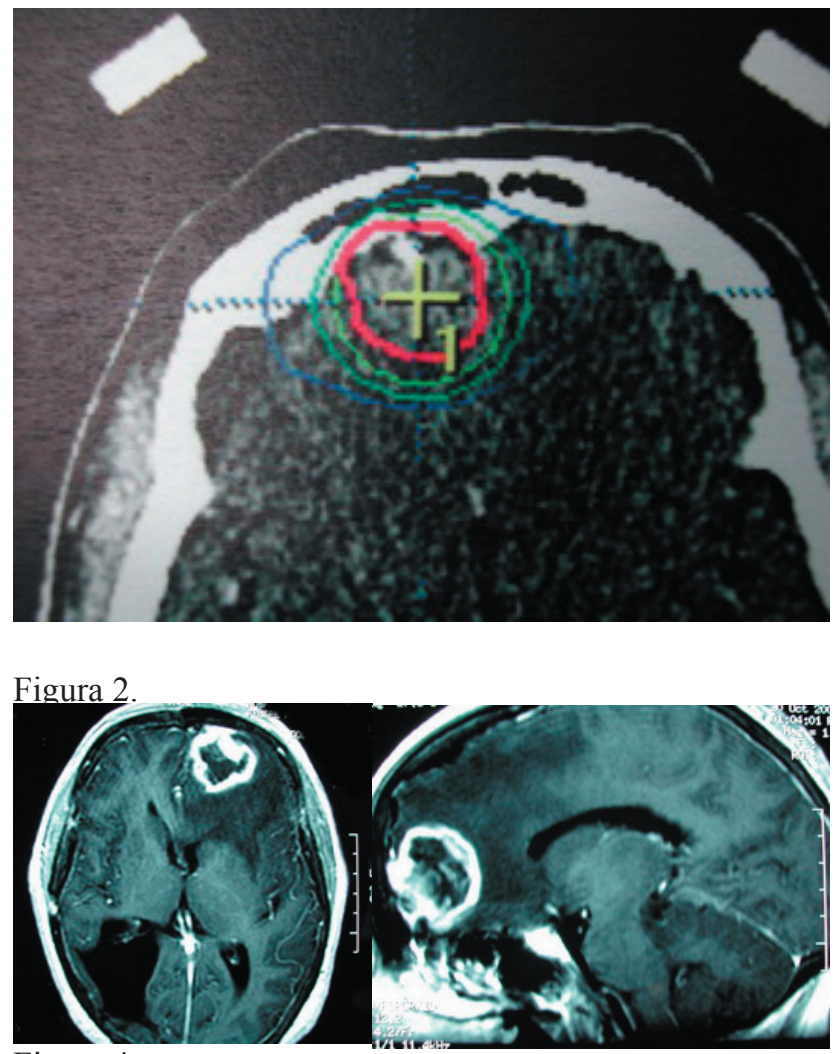

Figura 4.

de cobertura (isodosis del 80\%): 14,4Gy. Los órganos de riesgo recibieron una radiación muy por debajo de la tolerancia generalmente admitida. La paciente fue dada de alta a su domicilio unas horas más tarde sin incidencia alguna. Dos meses más tarde (julio 06) la paciente comenzó con un cuadro de cefalea persistente, hemiparesia derecha y disfasia. Acudió a su hospital donde, tras TAC craneal, se evidenció una lesión irregular en polo frontal izquierdo, con importante edema perilesional y gran efecto masa sobre el sistema ventricular con desplazamiento de casi $10 \mathrm{~mm}$ de la línea media. Se inició tratamiento con dexametasona a dosis de $8 \mathrm{mg}$ cada 8 horas y disminución progresiva hasta retirada en 12 días. Sin embargo, la paciente no toleró este descenso terapéutico por ulterior empeoramiento de su focalidad neurológica e intensificación de la cefalea, debiéndose incrementar la dosis de esteroides de nuevo. Se realizaron RNM de control en agosto y septiembre (Fig. 3), observándose una importante zona, informada de radionecrosis frontal, con captación de Gd de forma abigarrada, de 30x30x40mm de diámetro máximos, rodeada de una amplia zona de edema cerebral que colapsaba por completo el sistema ventricular anterior y seguía desviando la línea media en igual magnitud.

En Octubre se realizó nueva RNM cuya imagen fue idéntica a la de Agosto y Septiembre, a pesar del prolongado tratamiento con esteroides (Fig. 4). 

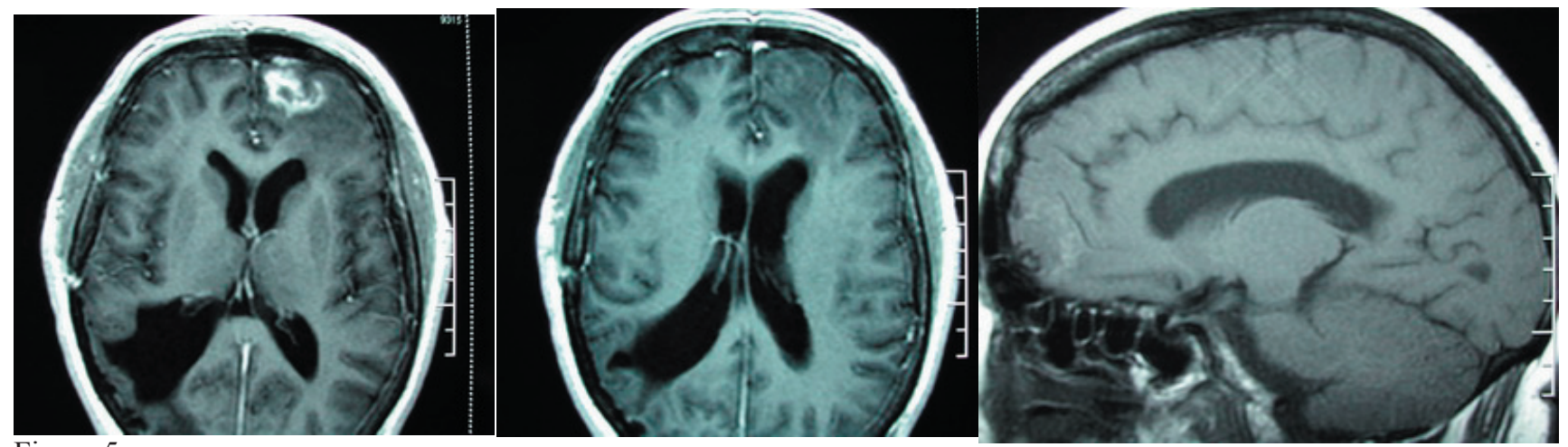

Figura 5.

En tales circunstancias se inició tratamiento con oxígeno al $100 \%$ a 2,5 atmósferas absolutas, en sesiones de 60 minutos al día. Se añadió al tratamiento enoxaparina sódica en dosis de 40mg al día en inyección subcutánea. Al haber transcurrido aproximadamente 20 sesiones, la paciente experimentó una progresiva mejoría de su cuadro clínico, lo que permitió rebajar la dosis de esteroides hasta suprimirlos por completo diez días más tarde. Tras completar un total de 40 sesiones, la paciente se encuentra asintomática. Una nueva RNM de control en Enero 07 (Fig. 5) ha puesto de manifiesto una casi completa desaparición del edema cerebral con restauración total de la línea media y del sistema ventricular. La zona radiada muestra todavía un aspecto abigarrado que sigue captando irregu- larmente $\mathrm{Gd}$, especialmente en su periferia, pero cuyos diámetros máximos se han reducido hasta 22x 20x18mm, siendo de difícil distinción el original meningioma objeto del tratamiento.

\section{Caso $n^{\circ} 2$}

Mujer de 76 años tratada en Junio 06 de un meningioma del peñasco derecho en ángulo pontocerebeloso cuyo volumen es de 4,24cc, con los siguientes parámetros: isocentros: 1; arcos: 6; colimador: $25 \mathrm{~mm}$; dosis de prescripción al isocentro: $14 \mathrm{~Gy}$; dosis de cobertura (isodosis del $85 \%$ ): 12Gy. El 10\% del tronco cerebral recibió 2,4Gy. El resto de órganos de riesgo no recibió radiación cuantificable alguna.

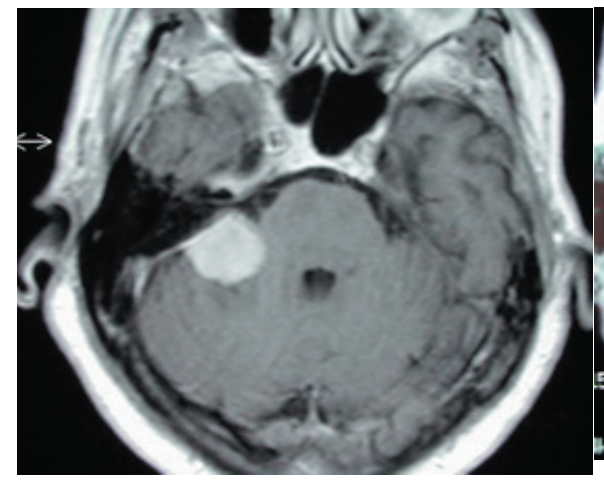

Caso 2

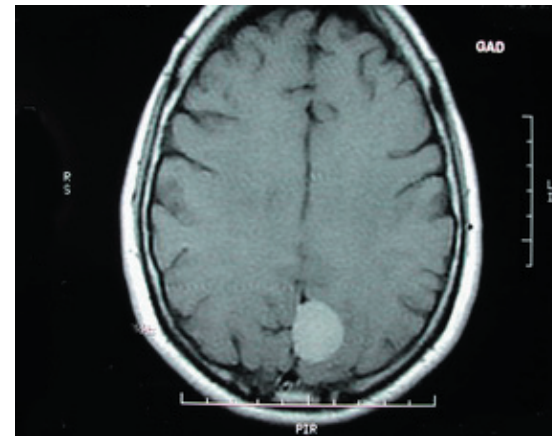

Caso 3

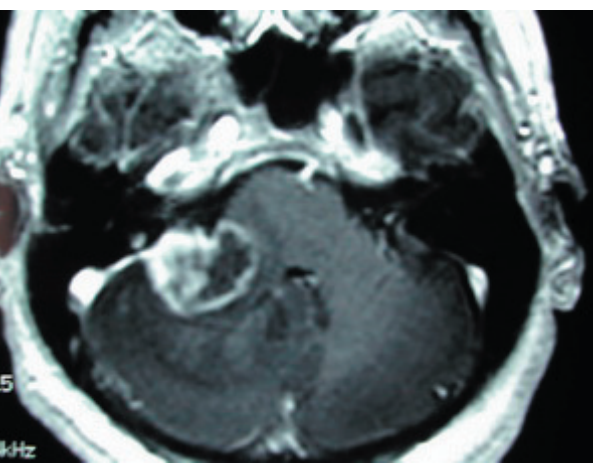

8 meses después

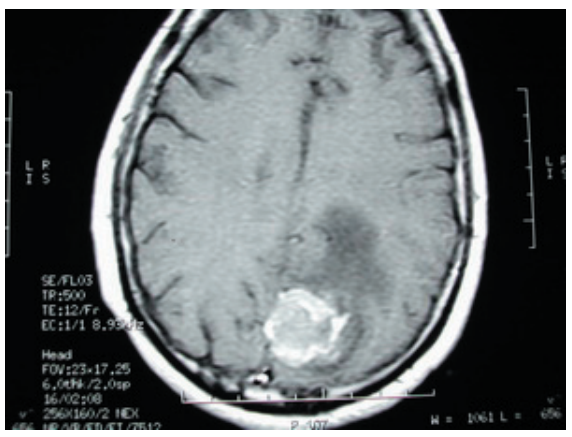

8 meses después

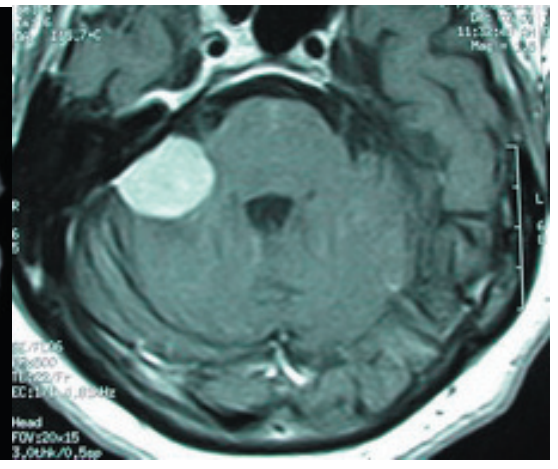

3 meses despúes de 40 sesiones de $\mathrm{O} 2$

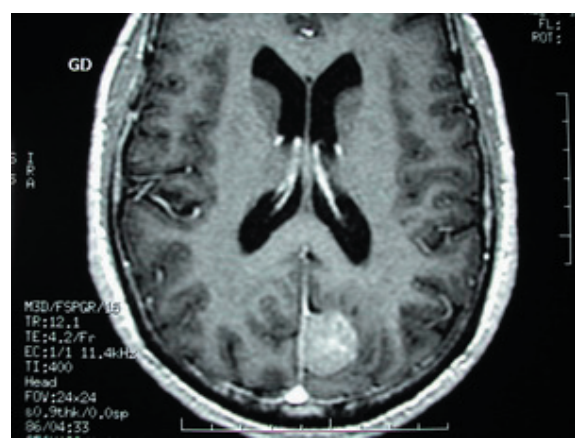

5 meses despúes de 40 sesiones de $\mathrm{O} 2$ 


\section{Caso $n^{\circ} 3$}

Mujer de 64 años con un meningioma parasagital parietooccipital izquierdo de $6,26 \mathrm{cc}$ de volumen, tratada en Febrero del 07 con 17,5Gy de prescripción a un único isocentro. Se usaron 6 arcos, colimador de $25 \mathrm{~mm}$ y la dosis de cobertura fue de $14 \mathrm{~Gy}$ en la isodosis del $80 \%$. Los órganos de riesgo no recibieron radiación cuantificable alguna.

Estos dos últimos casos tuvieron una evolución clínica similar a la experimentada en el caso $\mathrm{n}^{\mathrm{o}} 1$ : tras unos meses de estabilidad clínica post-radiocirugía, mostraron un empeoramiento que traducía un síndrome de hipertensión endocraneal, con predominio de ataxia en el meningioma del peñasco y de sensación de aturdimiento y cefalea en el parietooccipital. Los esteroides administrados no produjeron una mejoría sustancial. Tras la aplicación de $\mathrm{O} 2$ hiperbárico con el mismo régimen descrito en el primer caso, los cortejos sintomatológicos fueron mejorando en ambas pacientes y desaparecieron aun antes de completar el tratamiento prescrito de 40 sesiones. En la actualidad, ambas enfermas, al igual que la primera descrita, se hallan asintomáticas.

\section{Discusión}

El endotelio vascular es el sustrato principal donde intervienen diversos mecanismos tales como la alteración del factor de von Willebrand, la prostaciclina y el activador del plasminógeno, favoreciendo la agregación plaquetaria y ulteriormente los fenómenos trombóticos. Una supuesta "sincronización" de las células endoteliales transformaría estas células anormales y además aumentaría notablemente su población. Ello produciría una liberación anormal de citoquinas y poliaminas dando lugar a una alteración de la microcirculación y a una ruptura de la barrera hematoencefálica. La expresión genética, según el tipo de tejido irradiado (malformación vascular, tumor maligno o benigno, etc), puede jugar también un importante papel en el desarrollo de estos fenómenos ${ }^{17}$. Además de los recursos terapéuticos señalados más arriba, se siguen ensayando diversos inhibidores del factor activador de las plaquetas, tales como el BN52021 o la Difluorometilornitina, cuya vía terapéutica común sería el bloqueo o la eliminación de las poliaminas y citoquinas así como la potenciación del glutatión, elemento de efecto radioprotector, tendente a mantener o restaurar la integridad de la barrera hematoencefálica ${ }^{8,16}$.

En la literatura se siguen comunicando casos graves de radionecrosis cerebral, algunos de aparición muy tardía, como 13 años después de haber sido tratada la lesión, en este caso una malformación vascular que, al igual que el caso citado por Tandom, tuvo un fatal desenlaces. Otros casos similares han tenido un mejor pronóstico pero debiendo recurrir a la exéresis quirúrgica que, en ciertos casos, no logró restaurar la función clínica deteriorada en mayor o menor grado de forma permanente. Es interesante resaltar que ni la dosis, la citoarquitectura de la malformación, el tamaño de la misma, el volumen radiado, o incluso el tamaño de la zona necrótica resecada influyeron en el pronóstico clínico de muchos de estos $\operatorname{casos}^{13}$. En otras series los casos de radionecrosis pueden estar relacionados con una dosis excesiva de radiación o, como postulan sus autores, por afectar la radiación a un volumen excesivo de tejido cerebral circundante sano ${ }^{18}$. En otros casos sólo puede encontrarse una explicación aludiendo a una hipersensibilidad idiosincrásica del paciente; en este contexto se ha podido evidenciar una hipersensibilidad in vitro de los fibroblastos de algunos pacientes que habían desarrollado radionecrosis no letales, convirtiéndose este ensayo en la base de un posible test predictivo de este efecto adverso ${ }^{12}$. Más evidentes y comprensibles pueden ser otros factores de "riesgo", tales como la baja conformación de la planificación respecto de la lesión tratada, la falta de homogeneidad intralesional de la radiación administrada con profusión de puntos "calientes" y el volumen lesional. El continuo ajuste "a la baja" de la radiación administrada es una constante histórica de la radiocirugía, en un intento de conseguir equilibrar los resultados terapéuticos con la no aparición de efectos adversos por un exceso de radiación.

Entre los tratamientos propuestos para casos extremos, la cirugía sigue teniendo un relevante papel, si bien la administración de oxígeno hiperbárico en combinación con los esteroides y la heparina de bajo peso molecular es una terapéutica que va ganando adeptos de forma progresiva ${ }^{11}$. En ciertos casos se propone asociar terapia fotodinámica ${ }^{1}$ y no sólo para lesiones intracraneales sino para patología diversa en cabeza y cuello ${ }^{14}$, mandíbula ${ }^{2}$, y no sólo en adultos, sino también para tratar satisfactoriamente las secuelas radioinducidas en el tratamiento de diversos cánceres en la infancia ${ }^{3}$.

Los meningiomas benignos son una indicación en aumento del tratamiento radioquirúrgico. Sin embargo, también constituyen una patología propicia para el desarrollo de complicaciones, sobre todo edema cerebral y radionecrosis, llegando a afectar hasta un $25 \%$ de los casos tratados, si bien sólo un $9 \%$ cursó con sintomatología ${ }^{4}$. El edema peritumoral o la hinchazón aislada del tumor tras radiocirugía está descrito esporádicamente en meningiomas, pero su curso suele ser más benigno y han respondido bien al tratamiento con esteroides. Estos efectos se creen debidos más que a radionecrosis, a una hinchazón intracapsular del tumor debido a calentamiento tras la radiocirugía y a alteraciones vasculares peritumorales ${ }^{6,7}$. La única similitud con los casos que presentamos está en el edema perilesional, pero las características abigarradas de la lesión y su resistencia al tratamiento antiinflamatorio los diferencian notablemente. De igual manera no suele ser habitual, como en nuestros casos, la aparición de estas com- 
plicaciones post-radiocirugía de forma tan precoz, entre dos y ocho meses tras el tratamiento. Se han comunicado, como factores propicios para la radionecrosis, los tumores de localización en la fosa anterior, los parasagitales ${ }^{10}$, los que han recibido una dosis marginal mayor de $18 \mathrm{~Gy}^{9}$ aquéllos otros con edema previo a la radiocirugía, los de volumen superior a los $10 \mathrm{cc}$ y los que no han sido operados previamente, es decir, que fueron tratados primariamente con radiocirugía $\mathrm{a}^{15}$.

\section{Conclusión}

La terapia combinada con oxígeno hiperbárico en casos graves de edema o radionecrosis sintomáticos postradiocirugía debe ser considerada como tratamiento efectivo antes de indicar, como último recurso, la cirugía que, en muchos casos, puede dejar secuelas irreversibles.

\section{Bibliografía}

1. Al-Waili, N.S., Butler, G.J., Beale, J., et al.: Hyperbaric oxygen and malignancies:a potential role in radiotherapy, chemotherapy, tumor surgery and phototherapy. Med Sci Monit 2005; 11: 279-289.

2. Annane, D., Depondt, J., Aubert, P., et al.: Hyperbaric oxygen therapy for radionecrosis of the jaw: a randomized, placebo-controlled, double-blind trial fron the ORN96 study group. J Clin Oncol 2004; 22: 4893-4900.

3. Ashamalla, H.L., Thom, S.R., Goldwein, J.W.: Hyperbaric oxygen therapy for the treatment of radiation-induced sequelae in children. The University of Pennsylvania experience. Cancer 1996; 77: 2407-2412.

4. Chang, J.H., Chang, J.W., Choi, J.Y., et al.: Complications after gamma knife radiosurgery for benign meningiomas. J Neurol Neurosurg Psychiatry 2003; 74: 226-230.

5. Chen, H.I., Burnett, M.G., Huse, J.T., et al.: Recurrent late cerebral necrosis with aggressive characteristics after radiosurgical treatment of an arteriovenous malformation. J Neurosurg 2006; 105: 455-460.

6. El Shehaby, A.: Mechanisms of edema after gamma knife surgery for meningiomas. J Neurosurg 2005; (Suppl) 102: 1-3.

7. El Shehaby, A.: Temporary symptomatic swelling of meningiomas after gamma knife surgery: report of two cases. J Neurosurg 2005; (Suppl) 102: 293-296.

8. Fike, J.R., Gobbel, G.T., Chou, D., et al.: Cellular proliferation and infiltration following insterstitial irradia- tion of normal dog brain is altered by an inhibitor of polyamine synthesis. Int J Radiat Oncol Biol Phys 1995; 32: 1035-1045.

9. Ganz, J.C., Schrottner, O., Pendl, G.: Radiation-induced edema after gamma knife treatment for meningiomas. Stereot Funct Neurosurg 1996; (Suppl)1, 66: 129-133.

10. Kalapurakal, J.A., Silverman, C.L., Akhtar, N., et al.: Intracranial meningiomas: factors that influence the development ef cerebrl edema after stereotactic radiosurgery and radiation therapy. Radiolgy 1997; 204: 461-465.

11. Leber, K.A., Eder, H.G., Kovac, H., et al.: Treatment of cerebral radionecrosis by hyperbaric oxygen therapy. Stereot Funct Neurosurg 1998; (Suppl)1, 70: 229-236.

12. Malone, S., Raaphorst, G.P., Gray, R., et al.: Enhanced in vitro radiosensitivity of skins fibroblasts in two patients developing brain necrosis following AVM radiosurgery: a new risk factor with potential for a predictive assay. Int J Radiat Oncol Biol Phys 2000; 47: 185-189.

13. Massengale, J.L., Levy, R.P., Marcellus, M., et al.: Outcomes of surgery for resection of symptomatic radiation injury after stereotactic radiosurgery for arteriovenous malformations. Neurosurgery 2006; 59: 553-560.

14. Narozny, W., Sicko, Z., Kot, J., et al.: Hyperbaric oxygen therapy in the treatment of complications of irradiation in head and neck area. Undersea Hyperb Med 2005; 32: 103-110.

15. Novotný, J., Kollová, A., Liscák, R.: Prediction of intracraneal edema alter radiosurgery of meningiomas. J Neurosurg 2006; (Suppl) 105: 120-126.

16. Schmitz, M.P., Combs, D.J., Dempsey, R.J.: Difluoromethylornithine decreases postischemic brain edema and blood-brain breakdown. Neurosurgery 1993;33: 882-888.

17. Tandom, N., Vollmer, D.G., New, P.Z., et al.: Fulminant radiation-induced necrosis after stereotactic radiation therapy to the posterior fossa. Case report and review of the literature. J Neurosurg 2001; 95: 507-512.

18. Valéry, C.A., Cornu, P., Noel, G., et al.: Predictive factors of radiation necrosis after radiosurgery for cerebral metastases. Stereot Funct Neurosurg 2003; 81: 115-119.

Pérez-Espejo, M.A.; García-Fernández, R.; Tobarra-González, B.M.; Palma Copete, J.D.; González-López, A.; De la Fuente-Muñoz, I.; Salinas-Ramos, J.; Felipe-Murcia, M.; Martínez-Lage, J.F.; Fernández-Pérez, J.: Eficacia del oxígeno hiperbárico en el tratamiento de la radionecrosis y el edema cerebral sintomáticos tras radiocirugía con acelerador lineal. Neurocirugía 2009; 20: 449-453. 